\title{
Strong light-matter coupling in ultrathin double dielectric mirror GaN microcavities
}

\author{
K. Bejtka, ${ }^{1,2}$ F. Réveret, ${ }^{3}$ R. W. Martin, ${ }^{1,2, a)}$ P. R. Edwards, ${ }^{1}$ A. Vasson, ${ }^{3}$ J. Leymarie, ${ }^{3}$ \\ I. R. Sellers, ${ }^{2}$ J. Y. Duboz, ${ }^{2}$ M. Leroux ${ }^{2}$ and F. Semond ${ }^{2}$ \\ ${ }^{1}$ Department of Physics, SUPA, University of Strathclyde, Glasgow G4 ONG, United Kingdom \\ ${ }^{2}$ CRHEA-CNRS, Rue Bernard Gregory, Parc Sophia Antipolis, 06560 Valbonne, France \\ ${ }^{3}$ LASMEA, UMR 6602 UBP/CNRS, 24 Avenue des Landais, F-63177 Aubière Cedex, France
}

(Received 5 May 2008; accepted 21 May 2008; published online 17 June 2008)

\begin{abstract}
Strong light-matter coupling is demonstrated at low temperature in an ultrathin GaN microcavity fabricated using two silica/zirconia Bragg mirrors, in addition to a three-period epitaxial $(\mathrm{Al}, \mathrm{Ga}) \mathrm{N}$ mirror serving as an etch stop and assuring good quality of the overgrown GaN. The $\lambda / 2$ cavity is grown by molecular beam epitaxy on a Si substrate. Analysis of angle-resolved data reveal key features of the strong coupling regime in both reflectivity and transmission spectra at $5 \mathrm{~K}$ : anticrossing with a normal mode splitting of $43 \pm 2 \mathrm{meV}$ and $56 \pm 2 \mathrm{meV}$ for reflectivity and transmission, respectively, and narrowing of the lower polariton linewidth near resonance. (C) 2008 American Institute of Physics. [DOI: 10.1063/1.2944263]
\end{abstract}

Strong coupling in semiconductor microcavities (MCs) has recently been the subject of intensive studies. III-nitride materials offer great potential in this area, due to their large exciton binding energy and large optical oscillator strengths. ${ }^{1}$ Room temperature strong coupling in III nitrides has been demonstrated in MCs fabricated using lower epitaxial III-nitride distributed Bragg reflectors (DBRs), ${ }^{2,3}$ which by necessity have relatively narrow high-reflectivity stop bands, in combination with an upper dielectric DBR. Although there have been significant advances in the performance of III-nitride DBRs, difficulties remain due to extension of the cavity modes into the DBR and absorption, as well as to the narrow stop bands. Crack free AlInN-based mirrors have been demonstrated ${ }^{3}$ but crack formation remains an issue for AlGaN-based DBRs. Alternatively, MCs with two dielectric DBR mirrors do not suffer so greatly from the above limitations and offer the potential for higher finesse MCs, reduced cavity length, and broad stop bands. Their use for both mirrors decreases the effective cavity length and should lead to larger exciton-photon coupling. ${ }^{4}$

However, the inclusion of a second, lower dielectric DBR is challenging as it requires removal of the substrate in order to access the underside of the active region. ${ }^{5-10}$ This can be performed by laser lift-off from a sapphire substrate ${ }^{5,6}$ plasma etching of a $\mathrm{SiC}$ substrate, ${ }^{7}$ wet or dry etching of a silicon substrate, ${ }^{8,10}$ or combination of mechanical grinding, chemomechanical polishing, and dry etching of a GaN substrate. ${ }^{9}$ Strong coupling was not demonstrated in any of these double dielectric MCs. The approaches either require removal of the buffer layers and thinning of the GaN after substrate removal or growth of the active region directly on the substrate. These steps are complicated to perform, with limitations including nonideal surface quality, inhomogeneity, and variation in cavity thickness. It is essential to have accurate control of the cavity thickness in these resonant structures, and it is desirable to have a simpler and more reliable fabrication method.

\footnotetext{
${ }^{a)}$ Electronic mail: r.w.martin@strath.ac.uk.
}

In this paper, we report a double dielectric mirror $\mathrm{MC}$ where the cavity length is defined by the epitaxial growth alone, leading to the observation of strong light-matter coupling in both reflectivity and transmission. The active region is an ultrathin, half-wavelength $(\lambda / 2)$ layer of high quality $\mathrm{GaN}$, which is embedded between two dielectric mirrors. The III-nitride structure was grown by molecular beam epitaxy (RIBER Compact 21) on a three-period epitaxial AlN $/ \mathrm{Al}_{0.2} \mathrm{Ga}_{0.8} \mathrm{~N}$ DBR grown directly on (111)-oriented silicon. Details of the growth of (0001)-oriented nitrides on silicon are described elsewhere. ${ }^{11}$ The short $\mathrm{Al}(\mathrm{Ga}) \mathrm{N}$ mirror permits the growth of good quality GaN very close to the $\mathrm{Si}$ substrate and will also subsequently serve as an etch stop. The small number of repeat periods in the DBR is chosen as a trade off, ensuring reasonable crystalline quality in the active region without crack formation. No cracks were visible in the resulting structure.

The MC structure is completed using two dielectric DBRs, comprised of $\mathrm{SiO}_{2} / \mathrm{ZrO}_{2}$ quarter-wavelength layers deposited by electron beam evaporation. The centers of the stop bands are set at a wavelength at $359 \mathrm{~nm}$, closely matched to the GaN excitonic emission. The first DBR, with seven pairs of $\mathrm{SiO}_{2} / \mathrm{ZrO}_{2}$, was deposited on top of the active MC layers following the epitaxial growth and the second onto the lower surface of the epitaxial mirror after its exposure by removal of the Si substrate using the following process. The top surface of the dielectric mirror-coated epitaxial structure was bonded to a sapphire carrier using UV-curable optical cement. The Si is then removed by combination of polishing and electron cyclotron resonance etching. The latter process will stop cleanly at the epitaxial interface. The $\mathrm{MC}$ is then completed by deposition of a three-period dielectric DBR on the exposed underside of the thin epitaxial mirror $(N$ face). Further details on the processing are given in Ref. 10, which reports weak coupling in a similar structure. In this work, the order of dielectrics in the final DBR was reversed to maintain a high index step at the semiconductordielectric interface.

The MC structure was characterised using angle resolved photoluminescence (PL), reflectivity (R), and trans- 


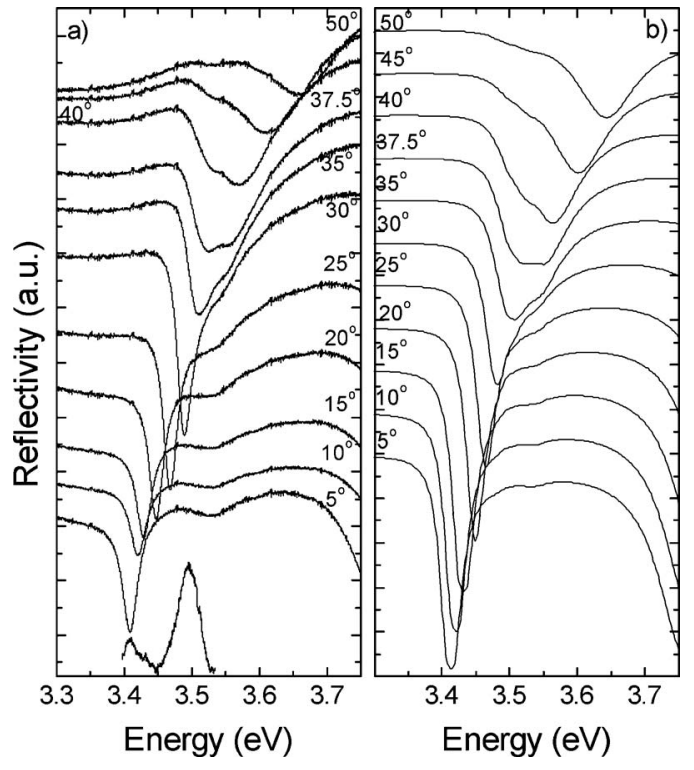

FIG. 1. (a) Experimental angle-resolved reflectivity spectra at $5 \mathrm{~K}$ in TE polarization. The lowest trace shows a PL spectrum at $5 \mathrm{~K}$. (b) Transfermatrix simulations at $5 \mathrm{~K}$.

mission (T) spectroscopy with the sample mounted inside a rotatable helium cryostat. The incident halogen lamp light is linearly polarized. The source and polarizer are fixed on a mobile rail with the same rotation axis as the cryostat allowing adjustment of the angle of incidence.

The measured TE-polarized reflectivity spectra of the $\mathrm{MC}$ for various angles of incidence from $5^{\circ}$ to $55^{\circ}$ at $5 \mathrm{~K}$ are shown in Fig. 1(a). Similar features were observed for TMpolarized measurements (not shown here) and this report will therefore describe only the TE-polarized measurements. The lowest trace in Fig. 1(a) shows a 5 K PL spectrum measured at an angle of incidence of $5^{\circ}$ in the same setup. The peak of the GaN PL occurs at an energy of $3.49 \mathrm{eV}$, which is high in comparison to the typical low temperature PL peak energy of $3.46 \mathrm{eV}$ for conventional micrometer thick $\mathrm{GaN}$ on silicon. ${ }^{11}$ This high emission energy results from the trade off between tensile strain due to growth on $\mathrm{Si}$ and the compressive strain resulting from the $(\mathrm{Al}, \mathrm{Ga}) \mathrm{N} / \mathrm{AlN} \mathrm{DBR} .{ }^{10}$ The difference in energy between the excitonic features in the reflectivity and PL spectra appears because the reflectivity spectra are dominated by free excitons while the $5 \mathrm{~K}$ PL corresponds to donor bound exciton recombination. ${ }^{12}$

At an angle of $5^{\circ}$, two features are clearly observed in the reflectivity spectrum. The optical mode can be observed at $\sim 3.41 \mathrm{eV}$, negatively detuned with respect to the excitonic feature observed at $3.53 \mathrm{eV}$. As the angle of incidence is increased, there is a clear anticrossing between the excitonic mode and the optical mode, reaching resonance for an angle near $37.5^{\circ}$. The excitonic feature remains clearly visible throughout the anticrossing region. The low angle photonic mode linewidth gives an estimate of the cavity quality factor $Q$ of 105 not far below that estimated by simple simulation of the reflectance of the empty cavity, with nominal layer thicknesses for the three DBRs $(Q \approx 140)$.

The reflectance spectra were modeled using a standard $(2 \times 2)$ transfer matrix method ${ }^{2}$ with refractive index values based on those reported in Ref. 13. The GaN excitonic feature was included in the simulation with an oscillator strength of $20000 \mathrm{meV}^{2}$, somewhat reduced from the value

Author complimentary copy. Redistribution subject to AIP

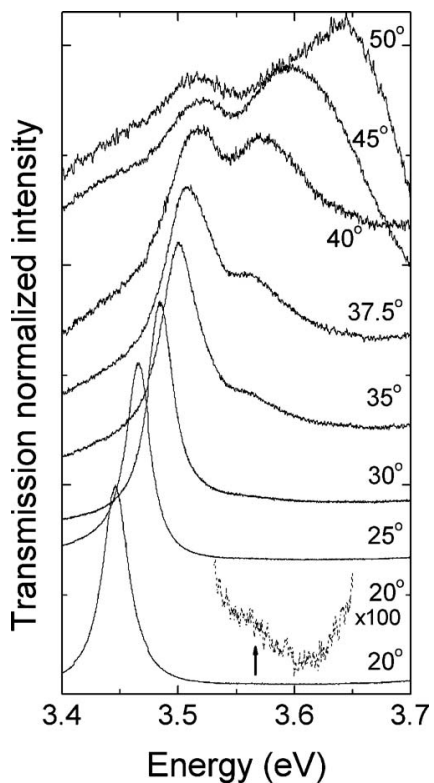

FIG. 2. Experimental angle-resolved transmission spectra at $5 \mathrm{~K}$ in $\mathrm{TE}$ polarization.

of $30000 \mathrm{meV}^{2}$ reported in Ref. 2, in order to obtain good agreement with our data. The result of the simulations is in excellent agreement with measurements, as shown in Fig. 1(b).

Strong coupling could also be resolved in the transmission spectra measured from the same sample, as shown in Fig. 2, for various angles of incidence (TE polarization) at $5 \mathrm{~K}$. Spectra for angles below $20^{\circ}$ were also recorded but are not plotted. At low angles of incidence $\left(<20^{\circ}\right)$, the photonlike mode $(Q \approx 125)$ is again negatively detuned with respect to the broad excitonlike transitions around $3.55 \mathrm{eV}$. The large negative detuning at this angle results in a relatively weak excitonic mode since the photonic contribution to this polariton mode is low and the measured transmission results only from the photons that leak from the MC. Nevertheless, the upper polariton branch can be followed at low angles in the transmission spectra, as shown for the expanded $20^{\circ}$ trace in Fig. 2.

The energies of the polariton modes determined from the reflectivity and transmission spectra are plotted as a function of angle in Fig. 3. Both clearly show the anticrossing asso-

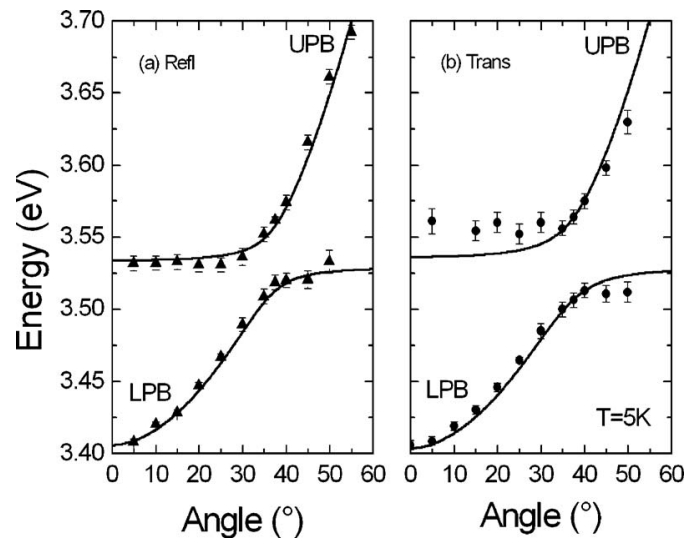

FIG. 3. Variation of the observed polariton mode energies with angle of incidence for (a) reflectivity and (b) transmission data. The solid lines plot the results of numerical simulation.

icense or copyright, see http://apl.aip.org/apl/copyright.jsp 


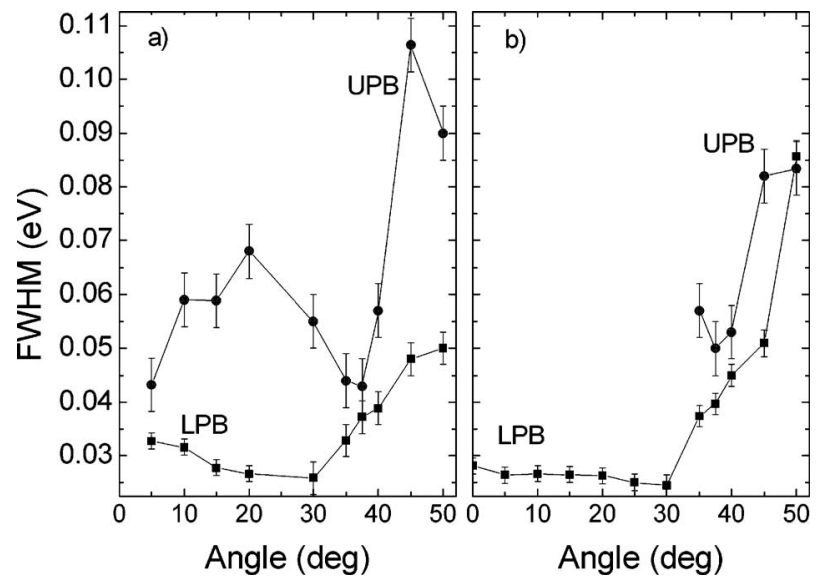

FIG. 4. Linewidth of the polariton branches as a function of angle of incidence for (a) reflectivity and (b) transmission data.

ciated with strong light-matter coupling. The Rabi energies estimated from the reflectivity and transmission data have values of $43 \pm 2 \mathrm{meV}$ and $56 \pm 2 \mathrm{meV}$, respectively, determined by the splitting of the upper and lower polariton branches (UPB, LPB) at resonance. Figure 3 also shows the results of the numerical simulation of the reflectivity and transmission data using the matrix analysis, reproducing the experimental data very well. The larger Rabi splitting observed in the transmission measurements compared with that from the reflectivity data follows the general observation that $\Omega_{\text {abs }}<\Omega_{\text {refl }}<\Omega_{\text {trans }}<\Omega_{\mathrm{qm}}$, where $\Omega_{\mathrm{qm}}$ is the quantummechanical cavity-polariton splitting. ${ }^{14}$ These data provide the first observation of strong coupling in transmission measurements on III-N MCs to the best of our knowledge.

Figure 4 plots the linewidth of the polariton modes measured from the reflectivity and transmission spectra. Where the features are well separated, they were fitted using a single Lorentzian (photon mode) or Gaussian (excitonic mode) and, where they overlap, two Gaussians were employed. Uncertainties remain due to the asymmetric nature of the UPB lineshapes caused mainly by coupling to continuum or excited states of the exciton and the increasing effects of absorption at higher energies. A notable feature of the data, with a magnitude exceeding the uncertainties, is the decrease in the LPB linewidth as the angle of incidence approaches resonance $\left(\approx 37.5^{\circ}\right)$. This linewidth narrowing is the result of the mixing of the photonic and excitonic modes in the strong coupling regime. The homogeneous linewidth $(\gamma)$ of the excitonic mode is the important parameter due to the large homogeneous lifetime. In this case (at $5 \mathrm{~K}$ ) $\gamma$ is very small $^{15}(<1.5 \mathrm{meV})$ and almost negligible in comparison to the excitonic inhomogeneous linewidth $(\sigma \approx 40 \mathrm{meV})$. Reference 16 gives the polariton linewidth as $\left(\sigma+\gamma_{\mathrm{ph}}\right) / 2$ for $\Omega_{\mathrm{abs}} \approx \sigma$, reducing to $\left(\gamma+\gamma_{\mathrm{ph}}\right) / 2$ for $\Omega_{\mathrm{Abs}} \gg \sigma$ where $\gamma_{\mathrm{ph}}$ is the linewidth of the photonic mode. The low angle spectra in Fig. 1 give an estimate of $\gamma_{\mathrm{ph}}$ as $32 \mathrm{meV}$, for which the two cases just described correspond to polariton linewidths of 16 and $36 \mathrm{meV}$, respectively. The measured LPB linewidth drops to about $25 \mathrm{meV}$ in the region of narrowing, lower than both $\gamma_{\mathrm{ph}}$ and $\frac{1}{2}\left(\sigma+\gamma_{\mathrm{ph}}\right)$ but not reaching $\frac{1}{2}\left(\gamma+\gamma_{\mathrm{ph}}\right)$.
This is in agreement with the measured Rabi splitting $\left(\Omega_{\text {trans }}>\sigma\right)$, intermediate between the two cases. In addition, absorption is already seen to be having an effect at angles below resonance, causing a broadening of the lines and masking the true minimum. The decrease of polariton linewidths near resonance for GaAs-based cavities has been reported earlier and shown to be a result of the inhomogeneous broadening of the excitons together with the interactions of the polaritons with disorder. ${ }^{17,18}$

In summary, we have demonstrated strong light-matter coupling at low temperature in both reflectivity and transmission spectra for an ultrathin bulk GaN MC structure with both upper and lower dielectric mirrors. The fabrication procedure involves removal of only the silicon substrate and the cavity length is controlled exclusively by epitaxy and not by processing as in earlier reports. Although the quality of the as grown material is still an issue, strong coupling is observed at low temperature with measured Rabi splittings of around $56 \mathrm{meV}$ and $43 \mathrm{meV}$ for transmission and reflectivity, respectively.

Funding from the EU projects STIMSCAT (STREP Contract No. 517769) and CLERMONT2 (MRTN-CT-2003503677) and from the UK EPSRC is acknowledged.

${ }^{1}$ A. Kavokin and B. Gil, Appl. Phys. Lett. 72, 2880 (1998).

${ }^{2}$ I. R. Sellers, F. Semond, M. Leroux, J. Massies, P. Disseix, A.-L. Henneghien, J. Leymarie, and A. Vasson, Phys. Rev. B 73, 033304 (2006); I. R. Sellers, F. Semond, M. Leroux, J. Massies, P. Disseix, A.-L. Henneghien, J. Leymarie, and A. Vasson, ibid. 74, 193308 (2006).

${ }^{3}$ R. Butté, G. Christmann, E. Feltin, J.-F. Carlin, M. Mosca, M. Ilegems, and N. Grandjean, Phys. Rev. B 73, 033315 (2006).

${ }^{4}$ H. Benisty, H. De Neve, and C. Weisbuch, IEEE J. Quantum Electron. 34, 1612 (1998).

${ }^{5}$ Y. K. Song, H. Zhou, M. Diagne, A. V. Nurmikko, R. P. Schneider, Jr., C. P. Kuo, M. R. Krames, R. S. Kern, C. Carter-Coman, and F. A. Kish, Appl. Phys. Lett. 76, 1662 (2000).

${ }^{6}$ R. W. Martin, P. R. Edwards, H.-S. Kim, K.-S. Kim, T. Kim, I. M. Watson, M. D. Dawson, Y. Cho, T. Sands, and N. W. Cheung, Appl. Phys. Lett. 79, 3029 (2001).

${ }^{7}$ T. Tawara, H. Gotoh, T. Akasaka, N. Kobayashi, and T. Saitoh, Appl. Phys. Lett. 83, 830 (2003).

${ }^{8}$ T. K. Kim, S. S. Yang, J. K. Hong, and G. M. Yang, Appl. Phys. Lett. 89, 041129 (2006).

${ }^{9}$ F. Rizzi, P. R. Edwards, K. Bejtka, F. Semond, X. N. Kang, G. Y. Zhang, E. Gu, M. D. Dawson, I. M. Watson, and R. W. Martin, Appl. Phys. Lett. 90, 111112 (2007).

${ }^{10}$ K. Bejtka, P. R. Edwards, R. W. Martin, F. Reveret, A. Vasson, J. Leymarie, I. R. Sellers, M. Leroux, and F. Semond, Semicond. Sci. Technol. 23, 045008 (2008).

${ }^{11}$ F. Semond, Y. Cordier, N. Grandjean, F. Natali, B. Damilano, S. Vézian, and J. Massies, Phys. Status Solidi A 188, 501 (2001).

${ }^{12}$ M. Leroux, N. Grandjean, B. Beaumont, G. Nataf, F. Semond, J. Massies, and P. Gibart, J. Appl. Phys. 86, 3721 (1999).

${ }^{13}$ N. Antoine-Vincent, F. Natali, M. Mihailovic, A. Vasson, J. Leymarie, P. Disseix, D. Byrne, F. Semond, and J. Massies, J. Appl. Phys. 93, 5222 (2003).

${ }^{14}$ R. P. Stanley, R. Houdré, C. Weisbuch, U. Oesterle, and M. Ilegems, Phys. Rev. B 53, 10995 (1996).

${ }^{15}$ O. Aoudé, P. Disseix, J. Leymarie, A. Vasson, M. Leroux, E. Aujol, B. Beaumont, A. Trassoudaine, and Y. André, Phys. Rev. B 77, 045206 (2008).

${ }^{16}$ R. Houdré, R. P. Stanley, and M. Ilegems, Phys. Rev. A 53, 2711 (1996).

${ }^{17}$ D. M. Whittaker, Phys. Rev. Lett. 80, 4791 (1998).

${ }^{18}$ A. V. Kavokin, Phys. Rev. B 57, 3757 (1998). 